\title{
Voice and the new corona virus
}

\author{
Magdalena Chirila ${ }^{1,2}$ \\ ${ }^{1}$ University of Medicine and Pharmacy of Cluj-Napoca \\ ${ }^{2}$ Iuliu Hatieganu University of Medicine and Pharmacy, Cluj-Napoca, Romania
}

April 28, 2020

\begin{abstract}
The new corona virus has become a global health concern. Voice has the potential to provide an easily obtained, non-invasive way to monitor physiological changes throughout the body. For health care providers with experience in the clinical management of patients with COVID-19 and other viral infections, including SARS and MERS, as well as sepsis and ARDS, the application of acoustical voice analysis should serve as a foundation for optimized supportive care to ensure the best possible chance for survival.
\end{abstract}

\section{What's known?}

The new corona virus has become a global health concern. Voice has the potential to provide an easily obtained, non-invasive way to monitor physiological changes throughout the body. Voice-related features were found to have a predictive value for different pathologies.

\section{What's new?}

A vocal test app on a smartphone could be used as a tool to identify patients most at risk of respiratory failure.

\section{Voice and the new corona virus}

The new corona virus has become a global health concern, as 1.537 .173 cases worldwide are declared today with 89.954 associated deaths.[1] Researchers around the world are looking for solutions for early detection, monitoring of the evolution of viral infection, etiological or pathophysiological treatment.

Voice is a unique human attribute. Voice has the potential to provide an easily obtained, non-invasive way to monitor physiological changes throughout the body. To produce the sounds, we need a system: the respiratory tract, the phonator, and the articulators. Respiration is necessary to produce the pressure and vibration: the air passes through the larynx, tissues vibrate to produce sound waves, and articulators are the shaping of raw sound into recognizable speech. Normal voice production depends on power and airflow supplied by the respiratory system. Any disturbance in one of the three subsystems of voice production may lead to a voice disorder. Recognizing associations among these factors, along with patient history, may help in identifying the possible causes of the voice disorder.[2]

Voice-related features were found to have a predictive value for different pathologies. A glottal-flow spectrum and vocal jitter were found to discriminate near-term risk suicidal adolescents.[6] Some studies have attempted to quantify voice parameters (acoustic amplitude, frequency variations), with the purpose of characterizing the Parkinson's disease dysphonic symptoms.[7] Wheezing and coughing, trouble breathing, coughing up mucus and shortness of breath are just a few chronic obstructive pulmonary disease (COPD) symptoms and many patients experience voice changes due to COPD. [8] In their pilot study, Murton et al.[9] 
analyzed the voices of heart failure (HF) patients as they underwent treatment for decompensated HF and returned to a stable clinical state.

How the voice acoustical analysis can help us identifying respiratory and breathing problems, the main concern today regarding patients with CoViD-19? Voice processing has become a fast-growing field. Voice disorder databases can be used in clinics as well as in automatic voice disorder detection systems to study the acoustic behavior of the voices. Audio recording is the most important basic requisite for voice quality assessment.[2] Coordination between the larynx and lower airways is essential for normal voice production. While most people with COVID-19 develop only mild or uncomplicated illness, approximately 14\% develop severe disease that requires hospitalization and oxygen support, and 5\% require admission to an ICU.[7] In severe cases, COVID-19 can be complicated by the acute respiratory distress syndrome (ARDS), sepsis and septic shock, multiorgan failure, including acute kidney injury and cardiac injury.[8] It may be possible to monitor a person's health remotely - using smartphones - by recording short speech samples and analyzing them for disease biomarkers. A vocal test app on a smartphone could be used as a tool to identify patients most at risk of respiratory failure. For health care providers with experience in the clinical management of patients with COVID-19 and other viral infections, including SARS and MERS, as well as sepsis and ARDS, the application of acoustical voice analysis should serve as a foundation for optimized supportive care to ensure the best possible chance for survival.

\section{Nothing to disclose.}

\section{References}

1. https://www.worldometers.info/coronavirus/

2. Dejonckere, P. H. (2010) Assessment of voice and respiratory function. In M. Remacle \& H. E. Eckel (Eds.), Surgery of larynx and trachea; pp. 11-26; New York, NY: Springer-Verlag Berlin Heidelberg.

3. Guidi A, Salvi S, Ottaviano M, Gentili C, Bertschy G, de Rossi D, Scilingo EP, Vanello N. (2015) Smartphone application for the analysis of prosodic features in running speech with a focus on bipolar disorders: system performance evaluateon and case study. Sensors; 15:28070-28087.

4. Tsuboi T, Watanabe H, Tanaka Y, Ohdake R, Hattori M, Kawabata K et al. (2017) Early detection of speech and voice disorders in Parkinsons's disease patients treated with sub thalamic nucleus deep brain stimulation: a 1-year follow-up study. J Neural Transm; 124:1547-1556.

5. Enas ME, Maghraby RA. (2014) Voice changes in patients with chronic obstructive pulmonary disease. Egypt J Chest Dis Tuberculosis; 63:561-567.

6. Murton OM, Hillman RE, Mehta DD. (2017) Acoustic speech analysis of patients with decompensated heart failure: a pilot study. J Acoust Soc Am; 142(4):EL401-EL407.

7. Team NCPERE. (2020) Vital surveillances: the epidemiological characteristics of an outbreak of 2019 novel coronavirus diseases (COVID-19) - China. China CDC Weekly; 2(8):113-122.

8. Yang X, Yu Y, Xu J, Shu H, Xia J, Liu H et al. (2020) Clinical course and outcomes of critically ill patients with SARS-CoV-2 pneumonia in Wuhan, China: a single-centered, retrospective, observational study. Lancet Respir Med. Epub 2020/02/28. doi: 10.1016/S2213-2600(20)30079-5. 